\title{
A Lotka-Volterra Competition Model with Cross-Diffusion
}

\author{
Wenyan Chen and Ya Chen \\ Department of Mathematics, Southeast University, Nanjing 210018, China \\ Correspondence should be addressed to Wenyan Chen; wychen@seu.edu.cn
}

Received 9 October 2012; Revised 24 January 2013; Accepted 29 January 2013

Academic Editor: Lan Xu

Copyright (c) 2013 W. Chen and Y. Chen. This is an open access article distributed under the Creative Commons Attribution License, which permits unrestricted use, distribution, and reproduction in any medium, provided the original work is properly cited.

A Lotka-Volterra competition model with cross-diffusions under homogeneous Dirichlet boundary condition is considered, where cross-diffusions are included in such a way that the two species run away from each other because of the competition between them. Using the method of upper and lower solutions, sufficient conditions for the existence of positive solutions are provided when the cross-diffusions are sufficiently small. Furthermore, the investigation of nonexistence of positive solutions is also presented.

\section{Introduction}

In this paper, we deal with the following Lotka-Volterra competition model with cross-diffusions:

$$
\begin{aligned}
-\Delta(u+\alpha v) & =u(a-u-c v), & x \in \Omega, \\
-\Delta(\beta u+v) & =v(b-v-d u), & x \in \Omega, \\
u=v & =0, \quad x \in \partial \Omega, &
\end{aligned}
$$

where $\Omega$ is a bounded domain in $\mathbf{R}^{N}$ with smooth boundary $\partial \Omega$ and all parameters $a, b, c, d, \alpha, \beta$ are positive constants. $u$ and $v$ stand for the densities of the two competitors; $a$ and $b$ are the intrinsic growth rates of $u$ and $v$, respectively; $c$ and $d$ are the competitive parameters between the two species; Here $\alpha$ and $\beta$ are referred to as cross-diffusions. Cross-diffusions express the two species run away from each other because of the competition between them. In this paper, the boundary condition is under homogeneous Dirichlet boundary condition which in biologically means that the boundary is not suitable for both species and they will all die on the boundary, and this is an ideal case.

In order to describe the meaning of cross-diffusions in this model (1) from the biological point, we give the general model with intrinsic diffusion and cross-diffusion:

$$
\begin{aligned}
& \frac{\partial u}{\partial t}=\operatorname{div}\left\{k_{11}(u, v) \nabla u+k_{12}(u, v) \nabla v\right\}+f(u, v), \\
& \frac{\partial v}{\partial t}=\operatorname{div}\left\{k_{21}(u, v) \nabla u+k_{22}(u, v) \nabla v\right\}+g(u, v),
\end{aligned}
$$

where $u$ and $v$ stand for the densities of the two species, intrinsic diffusion parameters $k_{11}(u, v), k_{22}(u, v)>0$, crossdiffusion parameters $k_{12}(u, v), k_{21}(u, v)$,

$$
\begin{gathered}
J_{u}=-\left\{k_{11}(u, v) \nabla u+k_{12}(u, v) \nabla v\right\}, \\
J_{v}=-\left\{k_{21}(u, v) \nabla u+k_{22}(u, v) \nabla v\right\}
\end{gathered}
$$

can be seen as the out-flux vector of $u$ and $v$ at $x$. The cross-diffusion parameters $k_{12}(u, v), k_{21}(u, v) \geq 0$ imply that the two competitors $u$ and $v$ diffuse in the direction of lower contrary of their competitor to avoid each other. $f(u, v), g(u, v)$ are response function and in this paper the classical Logistic Type is considered and $\alpha, \beta \geq 0$. More biological meaning of the system can be seen in [1-3].

The method of upper and lower solutions is a useful tool to study the existence of solutions of elliptic systems. However, there are many difficulties in investigating the existences of positive solutions of strongly coupled elliptic systems. Recently, by changing general strongly coupled elliptic systems into weakly coupled ones, the author in paper [4] gives the method to judge the solutions existence of elliptic systems by using the Schauder theorem. Furthermore, the method can be used to solve the existence of solutions of strongly coupled elliptic systems. In [5] Ko and Ryu 
investigate Lotka-Volterra prey-predator model with crossdiffusion:

$$
\begin{gathered}
-\Delta u=u\left(a_{1}-u-b_{12} v\right), \quad x \in \Omega, \\
-D \Delta u-\Delta v=v\left(a_{2}+b_{21} u-v\right), \quad x \in \Omega, \\
u=v=0, \quad x \in \partial \Omega .
\end{gathered}
$$

Here $D$ may be positive or negative. Using the developing method of upper and lower solutions in [4], the author gave a sufficient conditions for the existence of positive solutions to (4). Inspired by the paper [5], we investigate the existence and nonexistence of positive solutions to (1).

The main goal of this paper is to provide sufficient conditions for the existence of positive solutions to (1) when the cross-diffusions $\alpha$ and $\beta$ are small. More precisely, we have the following theorem. Let $\lambda_{1}>0$ be the principal eigenvalue of $-\Delta$ under homogeneous Dirichlet boundary condition. It is well known that the principal eigenfunction $\phi$ corresponding to $\lambda_{1}$ does not change sign in $\Omega$ and $\|\phi\|_{\infty}=1$.

Theorem 1. If $\min \{a-c b, b-d a\}>\lambda_{1}$, then there exist positive constants $\bar{\alpha}=\bar{\alpha}(a, b, c, d, \Omega), \bar{\beta}=\bar{\beta}(a, b, c, d, \Omega)$, when $\alpha<$ $\bar{\alpha}, \beta<\bar{\beta}$, (1) has at least one positive solution.

For $\alpha=\beta=0$, (1) is the Lotka-Volterra competition model under homogeneous Dirichlet boundary condition. In $[6,7]$, the authors use different methods to prove the existence of positive solutions, a sufficient condition for the existence is $\min \{a-c b, b-d a\}>\lambda_{1}$. The conclusion implies that weakly cross-diffusion does not affect the existence of positive solution.

This paper is organized as follows. In Section 2, the existence theorem of solutions for a general class of strongly coupled elliptic systems is presented using the method of upper and lower solutions. In Section 3, sufficient conditions for the existence and nonexistence of positive solutions of (1) are investigated. Moreover, we give the corresponding results simply if the competitive system only has one cross-diffusion.

\section{The Existence Theorem of Solutions for a Class of Strongly Coupled Elliptic Systems}

In this section, we presented the existence theorem of solutions for a general class of strongly coupled elliptic systems:

$$
\begin{gathered}
-\Delta A(u, v)=f_{1}(u, v), \quad x \in \Omega, \\
-\Delta B(u, v)=f_{2}(u, v), \quad x \in \Omega, \\
u=v=0, \quad x \in \partial \Omega .
\end{gathered}
$$

Here let $A, B, f_{1}, f_{2}$ satisfy the following hypotheses conditions.

(H1) $U, V$ are domain in $\mathbf{R}^{2},(0,0) \in U .(A, B)$ is a $C^{2}$ function about $(u, v)$ from $U$ to $V, A(0,0)=B(0,0)=$ 0 , and have a continuous inverse $\left(A^{*}, B^{*}\right) \in C^{2}(V, U)$. Then for all $(u, v) \in U$, let

$$
w=A(u, v), \quad z=B(u, v) .
$$

There exists only one $(w, z) \in V$, satisfying

$$
u=A^{*}(w, z), \quad v=B^{*}(w, z) .
$$

(H2) The function $A^{*}$ is increasing in $w$ and decreasing in $z ; B^{*}$ is decreasing in $w$ and increasing in $z$.

(H3) The functions $f_{1}(u, v), f_{2}(u, v)$ are Lipschitz continuous in $U$, and there exist positive constants $M_{1}, M_{2}$ such that for all $(u, v) \in U$, the function $f_{1}(u, v)+$ $M_{1} A(u, v)$ is increasing in $u$; the function $f_{2}(u, v)+$ $M_{2} B(u, v)$ is increasing in $v$.

According to the hypothesis ( $\mathrm{H1}$ ), (5) can be rewritten as the following equal PDE equations:

$$
\begin{gathered}
-\Delta w+M_{1} w=f_{1}(u, v)+M_{1} A(u, v), \quad x \in \Omega, \\
-\Delta z+M_{2} z=f_{2}(u, v)+M_{2} B(u, v), \quad x \in \Omega, \\
u=A^{*}(w, z), \quad v=B^{*}(w, z), \quad x \in \Omega, \\
w=z=0, \quad x \in \partial \Omega .
\end{gathered}
$$

Remark 2. According to the hypothesis (H1), (5) can also be equal to the following weakly coupled elliptic equations:

$$
\begin{array}{cc}
-\Delta w=f_{1}\left(A^{*}(w, z), B^{*}(w, z)\right):=g_{1}(w, z), & x \in \Omega, \\
-\Delta z=f_{2}\left(A^{*}(w, z), B^{*}(w, z)\right):=g_{2}(w, z), & x \in \Omega, \\
w=z=0, \quad x \in \partial \Omega . &
\end{array}
$$

In its pure form, (9) is simpler than (8). However, due to the complicity of mixed functions $g_{1}(w(x), z(x))$ and $g_{2}(w(x), z(x))$, it is difficult to find the solutions of (9) directly. Therefore, we discuss (8).

Assume functions $\bar{u}, \bar{v}, \underline{u}, \underline{v} \in C(\bar{\Omega}), \bar{w}, \bar{z}, \underline{w}, \underline{z} \quad \epsilon$ $C^{\alpha}(\bar{\Omega}) \bigcap C^{2}(\Omega)$, the values of functions $(\bar{u}, \bar{v})$ and $(\underline{u}, \bar{v})$ are in $V$ and the values of functions $(\bar{w}, \bar{z})$ and $(\underline{w}, \underline{z})$ are in $\bar{U}$. To describe easily, let

$$
\begin{aligned}
& U=\{u \in C(\bar{\Omega}): \underline{u}(x) \leq u(x) \leq \bar{u}(x)\}, \\
& V=\{u \in C(\bar{\Omega}): \underline{v}(x) \leq v(x) \leq \bar{v}(x)\} .
\end{aligned}
$$

According the definition of upper and lower solutions in [4] and conditions (H1)-(H3), we give the definition of upper and lower solutions of (5).

Definition 3. A pair of functions $((\bar{u}, \bar{v}, \bar{w}, \bar{z}),(\underline{u}, \underline{v}, \underline{w}, \underline{z}))$ are called upper and lower solutions of (9) provided that they 
satisfy the relation $(\bar{u}, \bar{v}, \bar{w}, \bar{z}) \geq(\underline{u}, \underline{v}, \underline{w}, \underline{z})$, and for all $(u, v) \in$ $U \times V$, satisfy the following inequalities:

$$
\begin{array}{cc}
-\Delta \bar{w}+M_{1} \bar{w} \geq f_{1}(\bar{u}, v)+M_{1} A(\bar{u}, v), \quad x \in \Omega, \\
-\Delta \bar{z}+M_{2} \bar{z} \geq f_{2}(u, \bar{v})+M_{2} B(u, \bar{v}), \quad x \in \Omega, \\
-\Delta \underline{w}+M_{1} \underline{w} \leq f_{1}(\underline{u}, v)+M_{1} A(\underline{u}, v), \quad x \in \Omega, \\
-\Delta \underline{z}+M_{2} \underline{z} \leq f_{2}(u, \underline{v})+M_{2} B(u, \underline{v}), \quad x \in \Omega, \\
\bar{u} \geq A^{*}(\bar{w}, \underline{z}), \quad \bar{v} \geq B^{*}(\underline{w}, \bar{z}), \quad x \in \Omega, \\
\underline{u} \leq A^{*}(\underline{w}, \bar{z}), \quad \underline{v} \leq B^{*}(\bar{w}, \underline{z}), \quad x \in \Omega, \\
\bar{w} \geq 0 \geq \underline{w}, \quad \bar{z} \geq 0 \geq \underline{z}, \quad x \in \partial \Omega .
\end{array}
$$
2.1].

We can have the following conclusion from [4, Theorem

Proposition 4. Assume that (8) has coupled upper and lower solutions $((\bar{u}, \bar{v}, \bar{w}, \bar{z}),(\underline{u}, \underline{v}, \underline{w}, \underline{z}))$, then there exists at least one solution $(u, v, w, z)$, satisfying the relation

$$
(\underline{u}, \underline{v}, \underline{w}, \underline{z}) \leq(u, v, w, z) \leq(\bar{u}, \bar{v}, \bar{w}, \bar{z})
$$

Furthermore, $(u, v)$ is the solution of (5).

Next, if $\bar{u}, \bar{v}, \underline{u}, \underline{v}$ satisfy

$$
\begin{array}{ll}
\bar{u}=A^{*}(\bar{w}, \underline{z}), & \bar{v}=B^{*}(\underline{w}, \bar{z}), \\
\underline{u}=A^{*}(\underline{w}, \bar{z}), & \underline{v}=B^{*}(\bar{w}, \underline{z}),
\end{array}
$$

then

$$
\begin{array}{ll}
\bar{w}=A(\bar{u}, \underline{v}), & \bar{z}=B(\underline{u}, \bar{v}), \\
\underline{w}=A(\underline{u}, \bar{v}), & \underline{z}=B(\bar{u}, \underline{v}),
\end{array}
$$

(11) can be rewritten as

$$
\begin{array}{ll}
-\Delta A(\bar{u}, \underline{v})+M_{1} A(\bar{u}, \underline{v}) \geq f_{1}(\bar{u}, v)+M_{1} A(\bar{u}, v), & x \in \Omega, \\
-\Delta B(\underline{u}, \bar{v})+M_{2} B(\underline{u}, \bar{v}) \geq f_{2}(u, \bar{v})+M_{2} B(u, \bar{v}), & x \in \Omega, \\
-\Delta A(\underline{u}, \bar{v})+M_{1} A(\underline{u}, \bar{v}) \leq f_{1}(\underline{u}, v)+M_{1} A(\underline{u}, v), & x \in \Omega, \\
-\Delta B(\bar{u}, \underline{v})+M_{2} B(\bar{u}, \underline{v}) \leq f_{2}(u, \underline{v})+M_{2} B(u, \underline{v}), & x \in \Omega, \\
A(\bar{u}, \underline{v}) \geq 0 \geq A(\underline{u}, \bar{v}), \quad B(\underline{u}, \bar{v}) \geq 0 \geq B(\bar{u}, \underline{v}), & x \in \partial \Omega .
\end{array}
$$

Synthetically, we have the following result.

Theorem 5. If there is a pair of functions $((\bar{u}, \bar{v}),(\underline{u}, \underline{v}))$, satisfying

$$
(\bar{u}, \bar{v}, A(\bar{u}, \underline{v}), B(\underline{u}, \bar{v})) \geq(\underline{u}, \underline{v}, A(\underline{u}, \bar{v}), B(\bar{u}, \underline{v})),
$$

and for all $(u, v) \in U \times V$, (15) is satisfied, then (5) has at least one solution $(u, v)$, satisfying the relation $(\underline{u}, \underline{v}) \leq(u, v) \leq$ $(\bar{u}, \bar{v})$.
To make sure the upper and lower solutions reasonable, we give the following two lemmas; more details can be found in $[8,9]$.

Lemma 6. If the functions $u, v \in C^{1}(\bar{\Omega})$ satisfy $\left.u\right|_{\partial \Omega}=\left.v\right|_{\partial \Omega}=$ $0,\left.u\right|_{\Omega}>0,\left.(\partial u / \partial v)\right|_{\partial \Omega}<0, v$ is the outer unit normal vector of $\partial \Omega$, then there exists positive constant $\varepsilon$, such that $u(x)>$ $\varepsilon v(x)$, for all $x \in \Omega$.

For the equation:

$$
\begin{gathered}
-\Delta u=u(a-u), \quad x \in \Omega, \\
u=0, \quad x \in \partial \Omega .
\end{gathered}
$$

Lemma 7. If $a>\lambda_{1}$, then (17) has a unique positive solution $\theta_{a}$ satisfying $\theta_{a} \leq a$. In addition, $\theta_{a}$ is increasing with respect to $a$.

\section{A Lotka-Volterra Competition Model with Two Cross-Diffusions}

In this section, the existence of positive solutions of (1) corresponding to $\alpha \geq 0, \beta \geq 0$, is investigated by applying Theorem 5 to prove Theorem 1 .

Proof. We seek some positive constants $R, K, \delta, R, K>\lambda_{1}$ sufficiently large and $\delta$ sufficiently small, Lemma 6 may guarantee the existence of $\theta_{R}$ and $\theta_{K}$. It can be easily known from Hopf boundary lemma:

$$
\frac{\partial \phi}{\partial v}(x)<0, \quad \frac{\partial \theta_{R}}{\partial v}(x)<0, \quad \frac{\partial \theta_{K}}{\partial v}(x)<0, \quad \forall x \in \partial \Omega .
$$

Observe that $\min \{a-c b, b-d a\}>\lambda_{1}$, using Lemma 7, we can have $R, K, \delta, a<R<\left(b-\lambda_{1}\right) / d, b<K<\left(a-\lambda_{1}\right) / c$, satisfying the following three conditions:

(i) $\delta \phi(x)<\theta_{R}(x), \delta \phi(x)<\theta_{K}(x)$, for all $x \in \Omega$;

(ii) $\left(\partial\left(\theta_{R}-\delta \phi\right) / \partial v\right)(x)<0,\left(\partial\left(\theta_{K}-\delta \phi\right) / \partial v\right)(x)<0$;

(iii) $\delta<\min \left\{a-\lambda_{1}-c K, b-\lambda_{1}-d R\right\}$.

Let $M_{1}=2 R+c K, \quad M_{2}=2 K+d R$. Using Lemma 7 again, there exist $\bar{\alpha}=\bar{\alpha}(a, b, c, d, \Omega)<1, \bar{\beta}=\bar{\beta}(a, b, c, d, \Omega)<1$, for all $(\rho, \tau) \in[0, \bar{\alpha}) \times[0, \bar{\beta})$, for all $x \in \Omega$, satisfying

(iv) $\theta_{R}-\delta \phi>\rho\left(\theta_{K}-\delta \phi\right), \theta_{K}-\delta \phi>\tau\left(\theta_{R}-\delta \phi\right)$;

(v) $(R-a) \theta_{R}>\rho\left[M_{1} \theta_{K}-\left(M_{1}+\lambda_{1}\right) \delta \phi\right],(K-b) \theta_{K}>$ $\tau\left[M_{2} \theta_{R}-\left(M_{2}+\lambda_{1}\right) \delta \phi\right] ;$

(vi) $\left(a-\lambda_{1}-\delta-c K\right) \delta \phi>\rho\left[\left(K+M_{1}-\theta_{K}\right) \theta_{K}-M_{1} \delta \phi\right]$;

(vii) $\left(b-\lambda_{1}-\delta-d R\right) \delta \phi>\tau\left[\left(R+M_{2}-\theta_{R}\right) \theta_{R}-M_{2} \delta \phi\right]$.

We will verify $\bar{\alpha}, \bar{\beta}$ satisfying Theorem 5 . Suppose that $(\alpha, \beta) \in[0, \bar{\alpha}) \times[0, \bar{\beta})$. Then we construct a pair of upper and lower solutions of the form

$$
(\bar{u}, \bar{v})=\left(\theta_{R}, \theta_{K}\right), \quad(\underline{u}, \underline{v})=(\delta \phi, \delta \phi),
$$


where $\delta$ satisfies conditions (i)-(iii). Let

$$
A(u, v)=u+\alpha v, \quad B(u, v)=\beta u+v .
$$

Then

$$
A^{*}(w, z)=\frac{w-\alpha z}{1-\alpha \beta}, \quad B^{*}(w, z)=\frac{z-\beta w}{1-\alpha \beta}
$$

By simply computing, (H1) and (H2) are satisfied, where $U=$ $[0, R] \times[0, K], \quad V=[0, R+\alpha K] \times[0, K+\beta R]$.

Note

$$
f_{1}(u, v)=u(a-u-c v), \quad f_{2}(u, v)=v(b-v-d u) .
$$

And for all $(u, v) \in U$, we have

$$
\begin{aligned}
{\left[f_{1}(u, v)+M_{1} A(u, v)\right]_{u} } & =a-2 u-c v+M_{1} \\
& \geq-2 R-c K+M_{1}=0, \\
{\left[f_{2}(u, v)+M_{2} B(u, v)\right]_{v} } & =b-2 v-d u+M_{2} \\
& \geq-2 K-d R+M_{2}=0 .
\end{aligned}
$$

So (H3) is satisfied; observer that $\left.\bar{u}\right|_{\partial \Omega}=\left.\bar{v}\right|_{\partial \Omega}=\left.\underline{u}\right|_{\partial \Omega}=$ $\left.\underline{v}\right|_{\partial \Omega}=0,(\bar{u}, \bar{v}) \geq(\underline{u}, \underline{v})$ and (iv) and (15) and the boundary conditions of (16) can be checked. Therefore, if we want to obtain the existence of solutions through [4, Theorem 2.1], we should only verify for all $(u, v) \in U \times V$,

$$
\begin{array}{ll}
-\Delta A(\bar{u}, \underline{v})+M_{1} A(\bar{u}, \underline{v}) \geq f_{1}(\bar{u}, v)+M_{1} A(\bar{u}, v), & x \in \Omega, \\
-\Delta B(\underline{u}, \bar{v})+M_{2} B(\underline{u}, \bar{v}) \geq f_{2}(u, \bar{v})+M_{2} B(u, \bar{v}), & x \in \Omega, \\
-\Delta A(\underline{u}, \bar{v})+M_{1} A(\underline{u}, \bar{v}) \leq f_{1}(\underline{u}, v)+M_{1} A(\underline{u}, v), & x \in \Omega, \\
-\Delta B(\bar{u}, \underline{v})+M_{2} B(\bar{u}, \underline{v}) \leq f_{2}(u, \underline{v})+M_{2} B(u, \underline{v}), & x \in \Omega .
\end{array}
$$

Because $f_{1}$ is decreasing in $v, f_{2}$ is decreasing in $u$, and $A(u, v)$ is increasing in $v, B(u, v)$ is increasing in $u$, only to verify the following inequations:

$$
\begin{array}{ll}
-\Delta A(\bar{u}, \underline{v})+M_{1} A(\bar{u}, \underline{v}) \geq f_{1}(\bar{u}, \underline{v})+M_{1} A(\bar{u}, \bar{v}), & x \in \Omega, \\
-\Delta B(\underline{u}, \bar{v})+M_{2} B(\underline{u}, \bar{v}) \geq f_{2}(\underline{u}, \bar{v})+M_{2} B(\bar{u}, \bar{v}), & x \in \Omega, \\
-\Delta A(\underline{u}, \bar{v})+M_{1} A(\underline{u}, \bar{v}) \leq f_{1}(\underline{u}, \bar{v})+M_{1} A(\underline{u}, \underline{v}), & x \in \Omega, \\
-\Delta B(\bar{u}, \underline{v})+M_{2} B(\bar{u}, \underline{v}) \leq f_{2}(\bar{u}, \underline{v})+M_{2} B(\underline{u}, \underline{v}), & x \in \Omega .
\end{array}
$$

It is easy to check (25) by (v), (vi), and (vii). So from [4, Theorem 2.1], (1) has a solution $(u, v)$, in addition $(\bar{u}, \bar{v}) \geq$ $(u, v) \geq(\underline{u}, \underline{v})>(0,0)$.

In the end, before investigating the nonexistence of positive solutions of (1), we give its priori bound of positive solutions.
Theorem 8. Any positive solutions $(u, v)$ of (1) have a priori bound; that is

$$
u(x) \leq \frac{b}{d}, \quad v(x) \leq \frac{a}{c}
$$

Proof. Let $w=u+\alpha v, z=\beta u+v$; then

$$
u=\frac{w-\alpha z}{1-\alpha \beta}, \quad v=\frac{z-\beta w}{1-\alpha \beta} .
$$

Equation (1) can be rewritten as

$$
\begin{gathered}
-\Delta w=\frac{w-\alpha z}{1-\alpha \beta}\left(a-\frac{w-\alpha z}{1-\alpha \beta}-c \frac{z-\beta w}{1-\alpha \beta}\right), \quad x \in \Omega, \\
-\Delta z=\frac{z-\beta w}{1-\alpha \beta}\left(b-\frac{z-\beta w}{1-\alpha \beta}-d \frac{w-\alpha z}{1-\alpha \beta}\right), \quad x \in \Omega, \\
(w, z)=(0,0), \quad x \in \partial \Omega .
\end{gathered}
$$

Since $(u, v)>(0,0)$, it easily follows that $w-\alpha z>0, z-\beta w>$ 0 . Assume that $z(x)$ attains its positive maximum at $x_{0} \in \Omega$, then

$$
\begin{gathered}
a(1-\alpha \beta)-w\left(x_{0}\right)+\alpha z\left(x_{0}\right)-c z\left(x_{0}\right)+c \beta w\left(x_{0}\right)>0 \\
a(1-\alpha \beta)-c z\left(x_{0}\right)+c \beta \alpha z\left(x_{0}\right)>0, \\
z(x) \leq z\left(x_{0}\right) \leq \frac{a}{c}
\end{gathered}
$$

so that

$$
v=z-\beta u \leq z\left(x_{0}\right) \leq \frac{a}{c} .
$$

Similarly, we can obtain the desired result

$$
u \leq \frac{b}{d}
$$

Theorem 9. If one of the following conditions:

(i) $b \leq a d, \lambda_{1} \geq(b+c \beta(b / d)) /(1-\alpha \beta)$;

(ii) $(1-(\alpha+\beta) / 2) \lambda_{1} \geq \max \{a, b\}$;

is satisfied, then (1) with $\alpha<\bar{\alpha}, \beta<\bar{\beta}$ has no positive solution.

Proof. Multiplying $u$ and $v$ to the first and second equations in (1), and integrating these equations on $\Omega$, we have

$$
\begin{aligned}
& \int_{\Omega}|\nabla u|^{2} d x+\alpha \int_{\Omega} \nabla u \nabla v d x=\int_{\Omega} u^{2}(a-u-c v) d x \\
& \alpha \int_{\Omega}|\nabla v|^{2} d x+\int_{\Omega} \nabla u \nabla v d x=\int_{\Omega} u v(a-u-c v) d x, \\
& \beta \int_{\Omega}|\nabla u|^{2} d x+\int_{\Omega} \nabla u \nabla v d x=\int_{\Omega} u v(b-v-d u) d x, \\
& \int_{\Omega}|\nabla v|^{2} d x+\beta \int_{\Omega} \nabla u \nabla v d x=\int_{\Omega} v^{2}(b-v-d u) d x .
\end{aligned}
$$


(i) Suppose, by contradiction that (1) has a positive solution $(u, v)$, then the second and fourth equations in (32) yield

$$
\begin{aligned}
\int_{\Omega} v^{3} d x & +\beta \int_{\Omega} u v(a-u) d x \\
= & -(1-\alpha \beta) \int_{\Omega}|\nabla v|^{2} d x+\beta \int_{\Omega} c u v^{2} d x \\
& +\int_{\Omega} v^{2}(b-d u) d x
\end{aligned}
$$

Since $u \leq b / d$ by Theorem 8 , the left-hand side of (33) must be positive. On the other hand, the Poincare inequality, $\|\nabla v\|_{L^{2}}^{2} \geq \lambda_{1}\|v\|_{L^{2}}^{2}$, for $v \in W_{2}^{1}(\Omega)$ and the given assumption shows the following contradiction:

$$
\begin{gathered}
-(1-\alpha \beta) \int_{\Omega}|\nabla v|^{2} d x+\beta \int_{\Omega} c u v^{2} d x+\int_{\Omega} v^{2}(b-d u) d x \\
\leq-\left[(1-\alpha \beta) \lambda_{1}-c \beta \frac{b}{d}-b\right] \int_{\Omega} v^{2} d x \leq 0 .
\end{gathered}
$$

(ii) A contraction argument is also used assuming that (1) has a positive solution $(u, v)$. Adding the first equation to the fourth equation, and then subtracting $a \int_{\Omega} u^{2} d x+b \int_{\Omega} v^{2} d x$ from the both sides, the following identity is obtained:

$$
\begin{aligned}
& \int_{\Omega}|\nabla u|^{2} d x+(\alpha+\beta) \int_{\Omega} \nabla u \nabla v d x \\
& +\int_{\Omega}|\nabla v|^{2} d x-a \int_{\Omega} u^{2} d x-b \int_{\Omega} v^{2} d x \\
& \quad=-\int_{\Omega} u^{2}(u+c v) d x-\int_{\Omega} v^{2}(v+d u) d x .
\end{aligned}
$$

Since $2 \nabla u \nabla v=|\nabla(u+v)|^{2}-|\nabla u|^{2}-|\nabla v|^{2}$ and $(1-(\alpha+$ B)/2) $\lambda_{1} \geq \max \{a, b\}$, the Poincare inequality shows that the left-hand side of (35) must be nonnegative, more precisely,

$$
\begin{aligned}
\int_{\Omega}|\nabla u|^{2} d x+(\alpha+\beta) \int_{\Omega} \nabla u \nabla v d x \\
\quad+\int_{\Omega}|\nabla v|^{2} d x-a \int_{\Omega} u^{2} d x-b \int_{\Omega} v^{2} d x \\
=\left(1-\frac{\alpha+\beta}{2}\right) \int_{\Omega}|\nabla u|^{2} d x+\frac{\alpha+\beta}{2} \int_{\Omega}|\nabla(u+v)|^{2} d x \\
\quad+\left(1-\frac{\alpha+\beta}{2}\right) \int_{\Omega}|\nabla v|^{2} d x-a \int_{\Omega} u^{2} d x-b \int_{\Omega} v^{2} d x \\
\geq \quad\left[\left(1-\frac{\alpha+\beta}{2}\right) \lambda_{1}-a\right] \int_{\Omega} u^{2} d x+\frac{\alpha+\beta}{2} \\
\quad \cdot \int_{\Omega}|\nabla(u+v)|^{2} d x+\left[\left(1-\frac{\alpha+\beta}{2}\right) \lambda_{1}-b\right] \int_{\Omega} v^{2} d x
\end{aligned}
$$

$$
\begin{aligned}
\geq & {\left[\left(1-\frac{\alpha+\beta}{2}\right) \lambda_{1}-a\right] \int_{\Omega} u^{2} d x } \\
& +\left[\left(1-\frac{\alpha+\beta}{2}\right) \lambda_{1}-b\right] \int_{\Omega} v^{2} d x \geq 0 .
\end{aligned}
$$

However, this results in a contradiction since the right-hand side of (35) is clearly strictly negative by the positivity of $u$ and $v$.

Remark 10. Before closing this section, more sufficient conditions of the nonexistence of positive solutions of (1) with $\alpha+\beta>0, \alpha \beta=0$ are investigated. Take $\alpha=0, \beta>0$ for example, then (1) may be reduced as

$$
\begin{gathered}
-\Delta u=u(a-u-c v), \quad x \in \Omega, \\
-\Delta(\beta u+v)=v(b-v-d u), \quad x \in \Omega, \\
(u, v)=(0,0), \quad x \in \partial \Omega .
\end{gathered}
$$

Using the same method, we can obtain that (37) has no positive solution, if one of the following conditions is satisfied:
(i) $\lambda_{1} \geq b+\beta c a$;
(ii) $\lambda_{1} \geq a$;
(iii) $(1-\beta / 2) \lambda_{1} \geq \max \{a, b\}$;
(iv) $c<1<a / b$ and $(1-d) / \beta \leq \lambda_{1} /(b+\beta a) \leq 1$.

\section{References}

[1] Y. Lou and W.-M. Ni, "Diffusion, self-diffusion and crossdiffusion," Journal of Differential Equations, vol. 131, no. 1, pp. 79-131, 1996.

[2] Z. Ling and M. Pedersen, "Coexistence of two species in a strongly coupled cooperative model," Mathematical and Computer Modelling, vol. 45, no. 3-4, pp. 371-377, 2007.

[3] Y. Lou and W.-M. Ni, "Diffusion vs cross-diffusion: an elliptic approach," Journal of Differential Equations, vol. 154, no. 1, pp. 157-190, 1999.

[4] C. V. Pao, "Strongly coupled elliptic systems and applications to Lotka-Volterra models with cross-diffusion," Nonlinear Analysis. Theory, Methods \& Applications A, vol. 60, no. 7, pp. 1197$1217,2005$.

[5] W. Ko and K. Ryu, "On a predator-prey system with cross diffusion representing the tendency of predators in the presence of prey species," Journal of Mathematical Analysis and Applications, vol. 341, no. 2, pp. 1133-1142, 2008.

[6] A. Leung, "Equilibria and stabilities for competing-species reaction-diffusion equations with Dirichlet boundary data," Journal of Mathematical Analysis and Applications, vol. 73, no. 1, pp. 204-218, 1980.

[7] N. Lakoš, "Existence of steady-state solutions for a onepredator-two-prey system," SIAM Journal on Mathematical Analysis, vol. 21, no. 3, pp. 647-659, 1990.

[8] L. Li, "Coexistence theorems of steady states for predator-prey interacting systems," Transactions of the American Mathematical Society, vol. 305, no. 1, pp. 143-166, 1988.

[9] C. V. Pao, Nonlinear Parabolic and Elliptic Equations, Plenum Press, New York, NY, USA, 1992. 


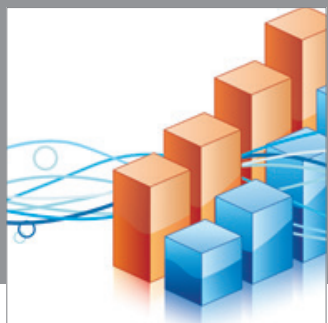

Advances in

Operations Research

mansans

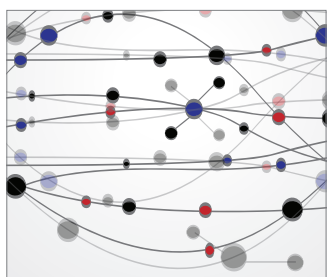

The Scientific World Journal
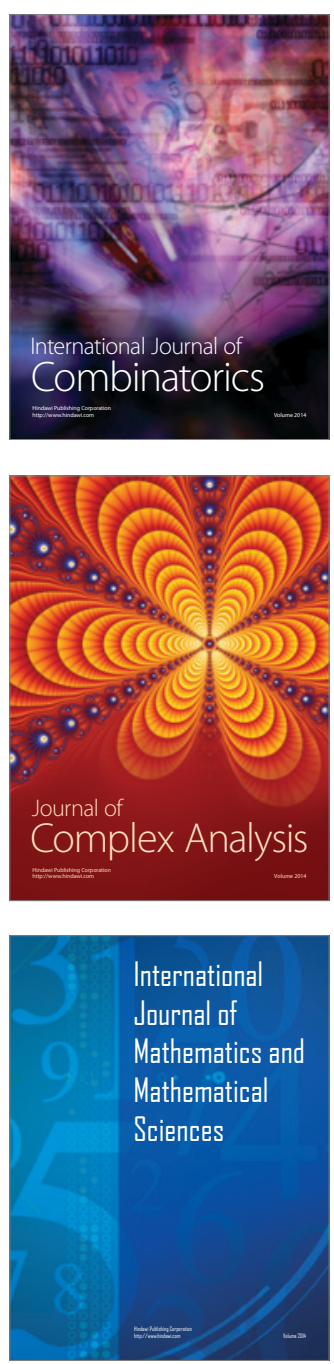
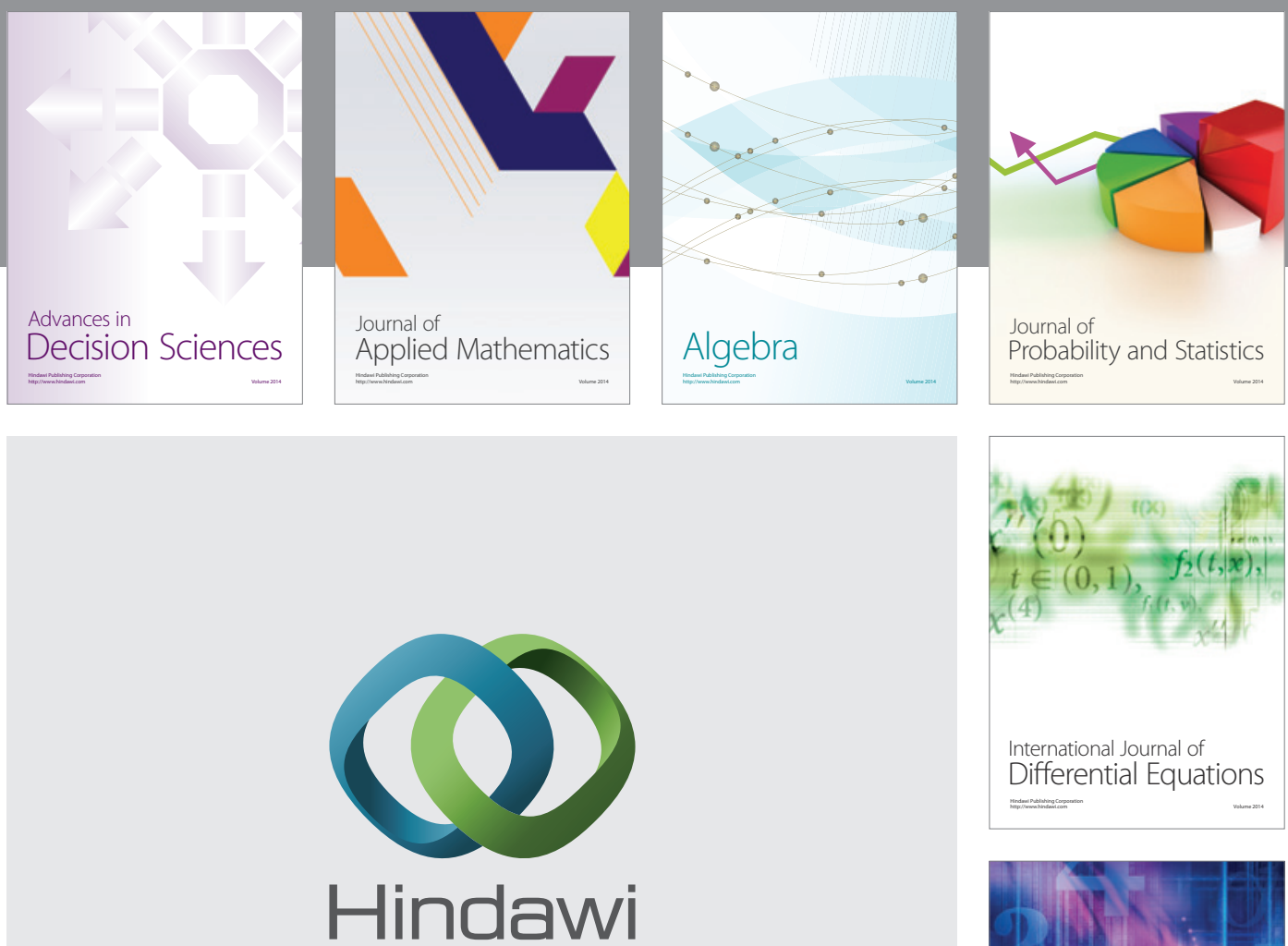

Submit your manuscripts at http://www.hindawi.com
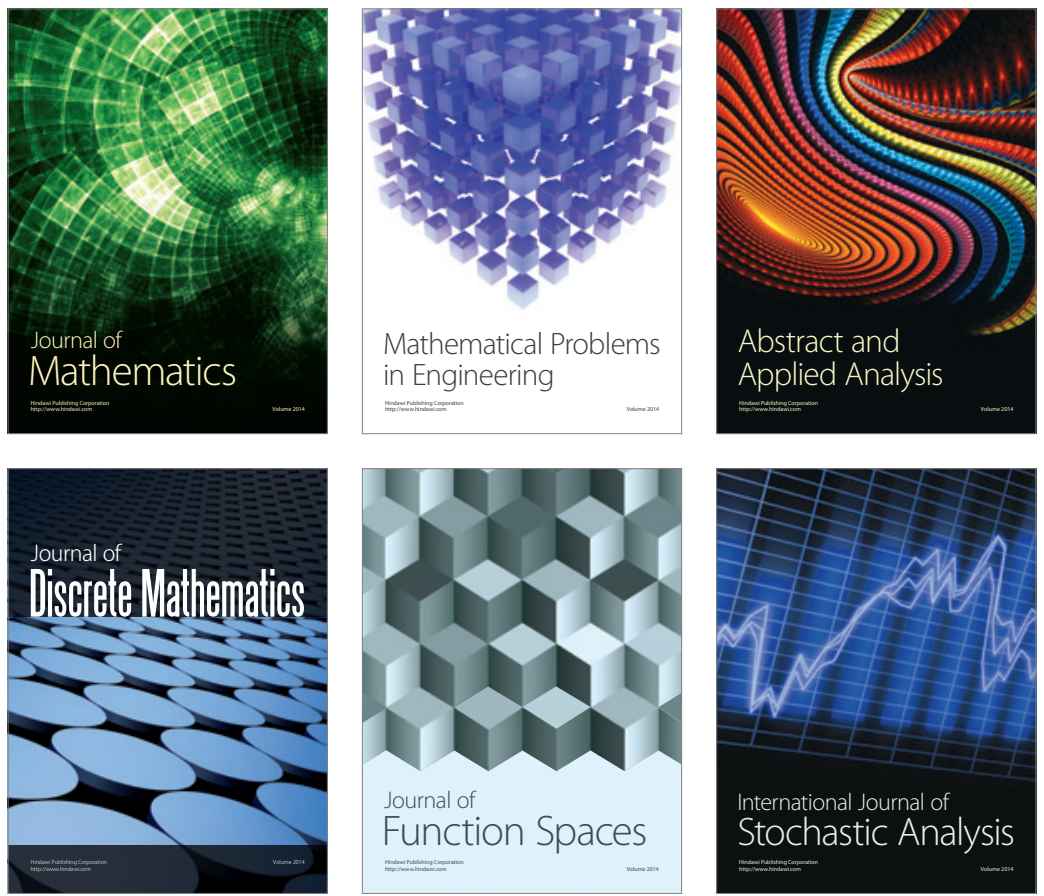

Journal of

Function Spaces

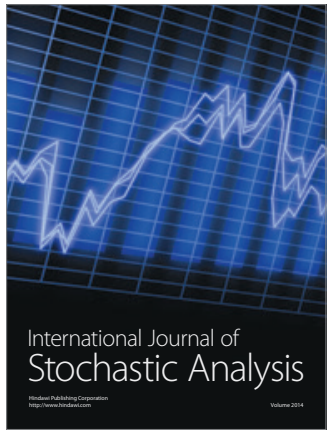

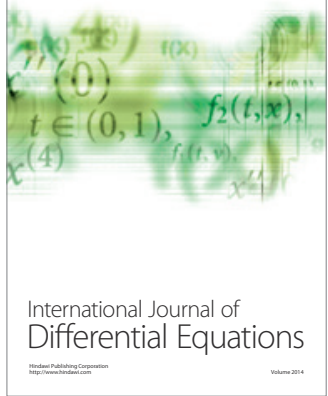
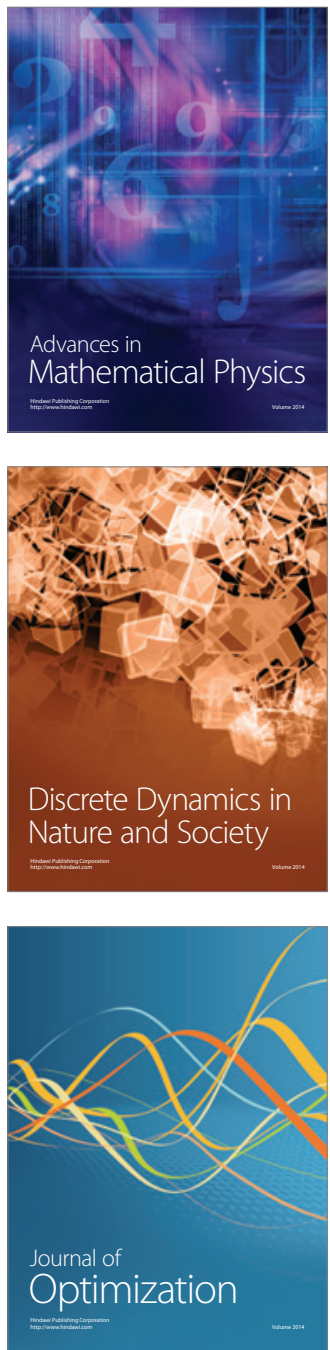